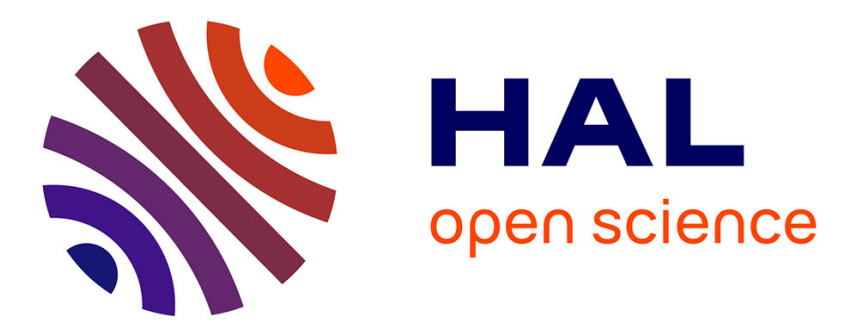

\title{
Ultrafast collisional dissipation of symmetric-top molecules probed by rotational alignment echoes
}

Junyang Ma, H Zhang, B Lavorel, F Billard, J Wu, C Boulet, Jean-Michel Hartmann, O Faucher

\section{- To cite this version:}

Junyang Ma, H Zhang, B Lavorel, F Billard, J Wu, et al.. Ultrafast collisional dissipation of symmetrictop molecules probed by rotational alignment echoes. Physical Review A : Atomic, molecular, and optical physics [1990-2015], 2020, 101 (4), pp.043417. 10.1103/PhysRevA.101.043417 . hal-02995743

\section{HAL Id: hal-02995743 \\ https://hal.science/hal-02995743}

Submitted on 9 Nov 2020

HAL is a multi-disciplinary open access archive for the deposit and dissemination of scientific research documents, whether they are published or not. The documents may come from teaching and research institutions in France or abroad, or from public or private research centers.
L'archive ouverte pluridisciplinaire HAL, est destinée au dépôt et à la diffusion de documents scientifiques de niveau recherche, publiés ou non, émanant des établissements d'enseignement et de recherche français ou étrangers, des laboratoires publics ou privés. 


\title{
Ultrafast collisional dissipation of symmetric-top molecules probed by rotational alignment echoes
}

\author{
Junyang Ma' ${ }^{1,2}$, H. Zhang', B. Lavorel ${ }^{1}$, F. Billard ${ }^{1}$, J. Wu ${ }^{2,3}$, C. Boulet ${ }^{4}$, J.-M. \\ Hartmann ${ }^{5}$, and O. Faucher ${ }^{1}$ \\ ${ }^{1}$ Laboratoire Interdisciplinaire CARNOT de Bourgogne, UMR 6303 CNRS-Université de \\ Bourgogne Franche-Comté, BP 47870, 21078 Dijon, France \\ ${ }^{2}$ State Key Laboratory of Precision Spectroscopy, East China Normal University, Shanghai \\ 200062, China \\ ${ }^{3}$ Collaborative Innovation Center of Extreme Optics, Shanxi University, Taiyuan, Shanxi 030006, \\ China \\ ${ }^{4}$ Institut des Sciences Moléculaires d'Orsay, CNRS, Université Paris-Sud, Université Paris-Saclay, \\ Orsay F-91405, France. \\ ${ }^{5}$ Laboratoire de Météorologie Dynamique/IPSL, CNRS, École polytechnique, Institut \\ polytechnique de Paris, Sorbonne Université, École Normale Supérieure, PSL Research \\ University, F-91120 Palaiseau, France
}

\begin{abstract}
We experimentally and theoretically investigate the ultrafast collisional dynamics of a symmetric-top molecule $\left(\mathrm{C}_{2} \mathrm{H}_{6}\right)$ in pure gas and mixtures with $\mathrm{He}$ at high density by employing the rotational alignment echo created by a pair of time-delayed intense laser kicks. The decrease of the amplitude of the echo when increasing the delay between the two laser pulses, reflecting the collisional relaxation of the system, is measured by probing the transient birefringence induced in the medium. The theoretical predictions, carried using purely classical molecular dynamics simulations, reproduce well the observed features, as demonstrated previously for a linear molecule. The analysis shows that the dissipation of the ethane alignment, despite the fact that this species has an extra rotational degree of freedom as compared to a linear molecule, barely involves more complex collisional relaxation channels due to
\end{abstract}


characteristics of the $\mathrm{C}_{2} \mathrm{H}_{6}-\mathrm{C}_{2} \mathrm{H}_{6}$ and $\mathrm{C}_{2} \mathrm{H}_{6}-\mathrm{He}$ interactions. However, our findings reveal that the dissipative dynamics of a symmetric-top molecule can be properly approached using the recently discovered rotational alignment echoes, which, so far, have been only tested for probing rotational decoherence of more simple (linear) molecules.

\section{Introduction}

Molecular dynamics in dissipative media, where interactions between atoms or molecules cause a rapid relaxation of the system, have attracted much attention for their important applications in many research fields [1-4]. Indeed, most real gas media being at non-negligible pressure, intermolecular collisions affect the spectral properties of radiative processes as well as the energy redistribution over the internal and external degrees of freedom of the system. In the past few years [5-10], quantum rotational revivals of molecular alignment induced by a single laser pulse have been successfully used to investigate the collisional relaxation in low-pressure gases. Unfortunately, this strategy cannot be used at high densities because the revivals vanish after a few tens of picoseconds due to the high rate of collisions. Recently, a new kind of echoes, called rotational alignment echoes [11], including full, high-order, fractional [12], rotated [13], and imaginary echoes [14], was discovered in gas molecules stimulated by a pair of time-delayed intense nonresonant femtosecond laser pulses [15-17]. In contrast to the rotational alignment revivals, whose temporal positions are fixed by the molecular rotational constant, the alignment echo offers much more flexibility as its time of appearance (at to twice the delay between the two laser pulses) is adjustable at will regardless of the molecule. It can hence be set much earlier than the first rotational alignment revival in order to probe relaxations occurring at very short time scales. Based on this phenomenon, a very recent study indeed demonstrated that the extremely fast collisional dissipation in linear molecular rotors at high pressures can be investigated in the few first picoseconds [18].

Time-resolved analyses of molecular collisional dynamics of aligned molecules 
have mainly focused on linear species [18-21] with only few investigations on symmetric-tops [22-24]. In particular, the approach using rotational alignment echoes has never been tested for nonlinear molecules. Using ethane $\left(\mathrm{C}_{2} \mathrm{H}_{6}\right)$ as a prototype, the present work fills this gap by investigating the collisional relaxation of a symmetric-top molecule at high-density, for pure gas and mixtures with He. Our results confirm that rotational alignment echoes provide a powerful tool for investigating ultrafast collisional relaxations from linear to symmetric-top molecules, and potentially in asymmetric-top molecules that exhibit more complex alignment features.

\section{Echo measurements}

Alignment echoes share many common properties with, for instance, photon echoes which result from the excitation of a quantum system by a pair of time delayed laser pulses. For rotational alignment echoes, a first ultrashort laser pulse produces a superposition of rotational coherences resulting in a slightly retarded alignment response of the molecular axes along the polarization of the field. Soon after this excitation, the alignment vanishes due to the free evolution of the coherences that oscillate at the different Raman frequencies excited by the large spectral bandwidth of the laser. A second ultrashort pulse, delayed by $\tau_{12}$, is then introduced in order to rephase most of the coherences of the system at the time $2 \tau_{12}$ where molecules realign as to form the echo. In many systems, it is usual to explain the echo phenomenon by invoking the time reversal mechanism; formally, the application of the second pulse reverses the course of time, so that the phase accumulated by the system during the first interval $\tau_{12}$ is compensated by the one accumulated during the second interval.

The experimental setup is schematically shown in Fig. 1. A pair of time-delayed $\left(\tau_{12}\right)$ collinear pump laser pulses $(100 \mathrm{fs}, 800 \mathrm{~nm}, 1 \mathrm{kHz})$ with parallel linear polarizations, labeled $\mathrm{P}_{1}$ and $\mathrm{P}_{2}$, are tightly focused into a high-pressure static cell. The probe beam (100 fs, $400 \mathrm{~nm}, 1 \mathrm{kHz})$, polarized at $45^{\circ}$ and temporally delayed from $\mathrm{P}_{1}$ by $\tau$, is spatially overlapped with the pump foci with a small crossing angle 
enabling to filter out the pump pulses after the interaction zone. The transient birefringence resulting from the anisotropic distribution of molecular orientations generated by the pumps is encoded in the modification of the polarization of the probe pulse, which is analyzed by a highly sensitive balanced detector consisting of a quarter-wave plate followed by a Wollaston prism (WP) and a pair of photodiodes. The separated vertical and horizontal polarization components after the WP are independently measured by the two photodiodes, which are connected head-to-tail so that the difference of their signals is directly obtained and amplified. All the measurements were performed at room temperature (295 K). More details about the experimental setup can be found in [18].

A measured temporal trace of the molecular alignment signal created by two successive nonresonant ultrashort laser pulses in pure $\mathrm{C}_{2} \mathrm{H}_{6}$ gas at low pressure is shown in Fig. 2(a). The laser pulse $P_{1}$ impulsively aligns some of the molecules along its polarization direction due to the anisotropy of the molecular polarizability. After a delay $\tau_{12}=3.2 \mathrm{ps}$, the pulse $\mathrm{P}_{2}$ is applied in order to produce the alignment echo at time $2 \tau_{12}=6.4$ ps after $P_{1}$. Its amplitude $S_{\text {echo }}$ is defined as the peak to dip change of the alignment signal. According to previous studies $[11,16,18]$, the sensitivity of the echo amplitude to the second pulse $\left(\mathrm{P}_{2}\right)$ intensity varies with the delay $\tau_{12}$. This feature originates from the fact that, in contrast with spin or photon echoes, alignment echoes take place in a multilevel system where the interference between many quantum pathways [16] leads to a partial rephasing of the coherences excited by the first pulse. A way to limit the experimental biases possibly resulting from the entanglement between $\mathrm{P}_{2}$ and $\tau_{12}$ is to find a compromised intensity of $\mathrm{P}_{2}$ for which $\mathrm{S}_{\text {echo varies little }}$ over a limited range of $\tau_{12}$ [18]. As shown by Fig. 2(b), theoretical predictions obtained by solving the time-dependent Schrödinger equation indicate that, for $\mathrm{P}_{2}$ set to $28 \mathrm{TW} / \mathrm{cm}^{2}, \mathrm{~S}_{\text {echo }}$ is almost constant over a $\sim 2$ ps wide range around $\tau_{12} \approx 3$ ps. Based on these simulations, we maximized $S_{\text {echo at }} \tau_{12} \sim 6$ ps by adjusting the intensity of the second pulse around $28 \mathrm{TW} / \mathrm{cm}^{2}$. This was done for pure $\mathrm{C}_{2} \mathrm{H}_{6}$ at a low pressure $(0.2$ bar $)$ in order to avoid any significant collision-induced decay of the 
echo amplitude. As shown in Fig. 2(b), the measured echo amplitude is indeed almost constant with less than $10 \%$ variations when $\tau_{12}$ is tuned between 2.5 and 4.5 ps. This range of delays and the intensity of $\mathrm{P}_{2}$ were thus retained to explore the collisional dissipation of the echo in pure $\mathrm{C}_{2} \mathrm{H}_{6}$ gases and $10 \% \mathrm{C}_{2} \mathrm{H}_{6}+90 \%$ He gas mixtures for total pressures up to of 14 and 19 bar, respectively.

Figure 3(a) presents the alignment signals recorded in a $\mathrm{C}_{2} \mathrm{H}_{6}-\mathrm{He}$ mixture for various delays $\tau_{12}$ and a pressure of 17 bar ( 15.4 amagat, 1 amagat corresponding to $\left.2.69 \times 10^{25} \mathrm{molec} / \mathrm{m}^{3}\right)$. As expected, the collisional dissipation induces the reduction, with increasing $\tau_{12}$, of the rotational alignment echo amplitude. The latter, plotted in Fig. 3(b), was first least-squares fitted by $A \exp \left[-2 \tau_{12} \gamma(d)\right]$, where $A$ defines the amplitude of the signal and $\gamma(d)$ is the decay rate at the chosen gas density $d$. Then, the density dependence of $\gamma(d)$ was fitted using the linear law $\gamma(d)=\gamma_{0} d$ resulting from the fact that, at the investigated densities, collisions are binary. The result of this exercise is shown in Figs. 4(a) and (b) which demonstrate the consistency of the measurements for both pure $\mathrm{C}_{2} \mathrm{H}_{6}$ and $\mathrm{C}_{2} \mathrm{H}_{6}-\mathrm{He}$ mixture. Finally, the density-normalized decay time constant of the rotational alignment echo was obtained from $\tau_{\mathrm{E}}=\gamma_{0}{ }^{-1}$, leading to the values summarized in Table I. For comparison, we also studied the decay time constant of the quantum alignment revivals of $\mathrm{C}_{2} \mathrm{H}_{6}$ measured in the $\mathrm{C}_{2} \mathrm{H}_{6}(10 \%)$-He gas mixtures and pure $\mathrm{C}_{2} \mathrm{H}_{6}$ gas. In that case, the dynamical alignment was produced by a single pulse $\left(\mathrm{P}_{1}\right)$ using the experimental setup depicted in Fig. 1. This study was conducted at low gas pressure (about 3 bar) so that the peak-to-peak amplitude of up to five revivals could be measured in the pure gas or the mixtures with $\mathrm{He}$ and fitted by an exponential law. The procedure to extract the related decay time constant is hence similar to that explained above for the determination of the echo decay time constant, provided that the series of values of $2 \tau_{12}$ is replaced by the times of appearance of the probed revivals (i.e. multiples of about 12.5 ps [22]). The time constant estimated for pure $\mathrm{C}_{2} \mathrm{H}_{6}$ and $\mathrm{C}_{2} \mathrm{H}_{6}$ infinitely diluted in He are reported in Table I and compared to the echo ones. Note that the value obtained for the revivals in a pure gas is similar to the one reported in Ref. [22]. 


\section{Theoretical analysis and discussion}

Calculations of the alignment signal in pure $\mathrm{C}_{2} \mathrm{H}_{6}$ and $\mathrm{C}_{2} \mathrm{H}_{6}-\mathrm{He}$ mixtures stimulated by two (for the echoes) and one (for the revivals) laser pulse(s) were made using molecular dynamics simulation (MDS). For the revivals, we used the requantized Classical MDS (rCMDS) described in [24] where all details on the computational procedure can be found. For the echoes, the same approach was used, except that the requantization procedure was not applied and purely Classical MDS (CMDS) were thus carried out. These choices were made in order to emphasize the fact that the revivals are of purely quantum nature while the echoes are a generic classical phenomenon, as demonstrated in the Supplementary Information of [25] (however note that, as for $\mathrm{N}_{2} \mathrm{O}$ [25], CMDS and rCMDS lead to very close results for the considered echoes in $\mathrm{C}_{2} \mathrm{H}_{6}$ ). As in our previous studies, the (r)CMDS enable to calculate the time evolution of the alignment factor under the conditions of the experiments (e.g. Fig. 3(a) and [24]), with one or two laser exciting laser pulses. These predictions were then treated, exactly as done with the measurements (see Sec. II), in order to retrieve the decay time constants of the revivals and echoes.

For pure $\mathrm{C}_{2} \mathrm{H}_{6}$, as in [24], the accurate ab initio intermolecular potential of [26] was used. In the absence of any available potential describing $\mathrm{C}_{2} \mathrm{H}_{6}-\mathrm{He}$ interactions, atom-atom 12-6 Lennard Jones (LJ) were used, with parameters $\varepsilon_{\mathrm{C}-\mathrm{He}}=33 \mathrm{~K}, \varepsilon_{\mathrm{H}-\mathrm{He}}=$ $7.4 \mathrm{~K}, \sigma_{\mathrm{C}-\mathrm{He}}=3.0 \AA$, and $\sigma_{\mathrm{H}-\mathrm{He}}=2.6 \AA$. These values were deduced from those associated with $\mathrm{C}-\mathrm{C}, \mathrm{H}-\mathrm{H}$, and $\mathrm{He}-\mathrm{He}$ pairs by using the (Lorentz-Berthelot) combination rules $\varepsilon_{\mathrm{X}-\mathrm{Y}}=\sqrt{\varepsilon_{\mathrm{X}-\mathrm{X}} \varepsilon_{\mathrm{Y}-\mathrm{Y}}}$ and $\sigma_{\mathrm{X}-\mathrm{Y}}=\left(\sigma_{\mathrm{X}-\mathrm{X}}+\sigma_{\mathrm{Y}-\mathrm{Y}}\right) / 2$. For the $\mathrm{C}-\mathrm{C}$ and $\mathrm{H}-\mathrm{H}$ parameters, we retained $\varepsilon_{\mathrm{C}-\mathrm{C}}=100 \mathrm{~K}, \varepsilon_{\mathrm{H}-\mathrm{H}}=5 \mathrm{~K}, \sigma_{\mathrm{C}-\mathrm{C}}=3.40 \AA$, and $\sigma_{\mathrm{H}-\mathrm{H}}=$ $2.65 \AA$, values that are consistent with those for the interaction between propane [27] and methane [28] molecule. For $\mathrm{He}-\mathrm{He}$, we used $\varepsilon_{\mathrm{He}-\mathrm{He}}=11 \mathrm{~K}$ and $\sigma_{\mathrm{He}-\mathrm{He}}=2.6 \AA$, obtained from a fit of the potential of [29] by a 12-6 LJ. The inset in Fig. 3(a) shows the echo signals predicted by the CMDS approach for a $\mathrm{C}_{2} \mathrm{H}_{6}-\mathrm{He}$ mixture at various delays $\tau_{12}$, whereas the corresponding amplitudes are displayed in Fig. 3(b). As can be seen by comparing the simulations to the experimental results for the same conditions 
displayed in Fig. 3, the agreement is very satisfactory. For comparison with the experimental data, three decay rates calculated at different densities are shown in Fig. 4 (a) and (b) for the gas mixture and pure $\mathrm{C}_{2} \mathrm{H}_{6}$, respectively. Notice that because collisions are binary, the CMDS results are strictly proportional to the density. As shown in Table I, the experimental results for the echoes are well predicted by the theory for both $\mathrm{C}_{2} \mathrm{H}_{6}-\mathrm{He}$ gas mixture and pure $\mathrm{C}_{2} \mathrm{H}_{6}$. It is observed that the experimental decay time constants of rotational alignment echoes and revivals, both well reproduced by the CMDS and rCMDS models, respectively, have close values. This trend is similar to those observed and predicted for pure $\mathrm{CO}_{2}$ and $\mathrm{CO}_{2}(4 \%)-\mathrm{He}$ [18]. In addition, for $\mathrm{C}_{2} \mathrm{H}_{6}$, collisions with $\mathrm{He}$ induce slower decays that when pure gas is considered, as observed and predicted previously for $\mathrm{CO}_{2}$ gas $[8,9]$. In both cases this results from the fact that, for $\mathrm{C}_{2} \mathrm{H}_{6}-\mathrm{He}$ (resp. $\mathrm{CO}_{2}-\mathrm{He}$ ), the interaction potential is at much shorter range and with a much shallower well when compared to the $\mathrm{C}_{2} \mathrm{H}_{6}-\mathrm{C}_{2} \mathrm{H}_{6}$ (resp. $\mathrm{CO}_{2}-\mathrm{CO}_{2}$ ) potential.

TABLE I: Measured and calculated collisional decay time constants in ps.amagat units for infinitely diluted $\mathrm{C}_{2} \mathrm{H}_{6}-\mathrm{He}$ gas mixture and pure $\mathrm{C}_{2} \mathrm{H}_{6}$ gas. For the measured values, the uncertainties correspond to two standard deviations.

\begin{tabular}{l|l|c|c}
\hline \multicolumn{2}{c|}{} & \multicolumn{1}{c}{ Experiment } & (r)CMDS model \\
\hline \multirow{2}{*}{$\mathrm{C}_{2} \mathrm{H}_{6}-\mathrm{He}$} & Revivals & $58.0 \pm 9.4$ & 63.0 \\
\cline { 2 - 4 } & Echo & $56.3 \pm 2.0$ & 59.0 \\
\hline \multirow{2}{*}{ Pure $\mathrm{C}_{2} \mathrm{H}_{6}$} & Revivals & $44.7 \pm 5.6$ & $44.9[24]$ \\
\cline { 2 - 4 } & Echo & $44.9 \pm 2.2$ & 42.9 \\
\hline
\end{tabular}

At this step, two points deserve discussion, which are: (i) Is the dissipation of the rotational alignment echoes (and revivals) in ethane gas influenced by the symmetric-top nature of this molecule (in other words is it different from that of a 
linear molecule)? (ii) What is the role of the nonsecular effects recently demonstrated [30] at the early stage of the relaxation of the alignment in $\mathrm{N}_{2} \mathrm{O}-\mathrm{He}$ mixtures?

The answer to the first question is no in the particular cases of pure $\mathrm{C}_{2} \mathrm{H}_{6}$ and $\mathrm{C}_{2} \mathrm{H}_{6}-\mathrm{He}$ mixtures. Indeed, as discussed in [24], the $\mathrm{C}_{2} \mathrm{H}_{6}-\mathrm{C}_{2} \mathrm{H}_{6}$ intermolecular potential [26] is almost insensitive to rotations of the molecules around their C-C bond. This implies that collisions do not significantly change the molecules' motion around this axis (i.e. they leave the quantum number $\mathrm{K}$ describing the rotation of the molecule around the $\mathrm{C}-\mathrm{C}$ axis unchanged, justifying the assumption made in [6]), so that collisional dissipation effects in pure ethane are very close to those occurring in a gas of linear molecules. For $\mathrm{C}_{2} \mathrm{H}_{6}$ colliding with $\mathrm{He}$, the situation is the same, since the forces applied to the $\mathrm{H}$ atoms by a nearby $\mathrm{He}$ are weak as a result of the very shallow $\mathrm{H}-\mathrm{He}$ potential well $\left(\varepsilon_{\mathrm{H}-\mathrm{He}}=7.4 \mathrm{~K}\right)$. As discussed in the conclusion of [24], finding a "good" molecular system to evidence the influence of the non-linearity of a symmetric-top molecule on the dissipation of its alignment is not easy. Indeed, not only the chosen molecule should have a large enough anisotropy of polarizability (in order to experimentally achieve a sufficient degree of alignment), but also its interaction with collision partners should significantly vary when the molecule is rotated around its symmetry axis and, in addition, an accurate intermolecular potential should be available.

In [30], it was experimentally and theoretically demonstrated that nonsecular effects resulting from collisional exchanges among the quantum coherences generated by the laser pulse slow down the pressure-induced decay of the echo in $\mathrm{N}_{2} \mathrm{O}-\mathrm{He}$ gas mixtures for short time delays. It is also very likely the case in $\mathrm{C}_{2} \mathrm{H}_{6}$, although the secular approximation should become valid sooner than in $\mathrm{N}_{2} \mathrm{O}$ since the latter has a smaller rotational constant (recall that nonsecular effects are significant at times such that the dephasing between significantly collisionally coupled coherences is smaller than a few times $\pi[30])$. In the present study, deviations from the secular behavior, which require studying the decay with density of the echo at fixed values of the delay $\tau_{12}$, cannot be evidenced since only the average decay over the time window of observation (i.e. the scanned range of delays between the two pulses) is studied. In 
any case, nonsecular effects, which are expected and likely present in the measurements, are included in the CMDS despite their classical nature, as demonstrated in [25]. Indeed, in the classical rotational phase space, they result from collisional transfers within the rotational speed filaments induced by the first pulse. Further studying this secular/nonsecular issue would require using the approach proposed in [30], i.e. to measure (and compute) the decay of the amplitude of each individual echo induced by pressure.

\section{Conclusion}

The ultrafast dissipation dynamics of rotational alignment echoes of the symmetric-top molecules $\mathrm{C}_{2} \mathrm{H}_{6}$ in high-pressure gases (pure and diluted in $\mathrm{He}$ ) have been experimentally and theoretically investigated. The decay time constant of the measured rotational alignment echoes (as that of the alignment revivals) is well reproduced by molecular dynamics simulations. These results, and the fact that the echoes and revivals show similar pressure-induced decay time constants, are in line with our previous work performed for the linear molecules $\mathrm{CO}_{2}$, and the analysis shows that ethane, due to some characteristics of the $\mathrm{C}_{2} \mathrm{H}_{6}-\mathrm{C}_{2} \mathrm{H}_{6}$ and $\mathrm{C}_{2} \mathrm{H}_{6}-\mathrm{He}$ interactions, practically behaves as a linear molecule. This study however corroborates the idea that rotational alignment echoes provide a relevant method for probing ultrafast rotational relaxation in high-density media where quantum alignment revivals become unusable [18] as well as when the quantum description of the system becomes intractable. The last applies to complex molecules, like asymmetric tops [7,31] and non-rigid molecules [32]. Since the echo is a classical phenomenon, its dissipation analyzed with the help of a classical model should in principle give access to the collisional relaxation of complex systems, including molecules embedded in helium nanodroplets [33,34].

Acknowledgments: The work was supported by the ERDF Operational Programme Burgundy 2014/2020 and the EIPHI Graduate School (contract "ANR-17-EURE-0002"). J. Ma acknowledges the support from the China Scholarship 
Council (CSC). J.-M. Hartmann benefited, for the computer simulations, from the IPSL mesocenter ESPRI facility which is supported by CNRS, UPMC, Labex L-IPSL, CNES, and Ecole Polytechnique. J.Wu acknowledges the support by the National Key R\&D Program (Grant No. 2018YFA0306303) and the NSFC (Grant Nos. 11425416, 11761141004, and 11834004). 


\section{References:}

[1] V. May, and O. Kuhn, Charge and Energy Transfer Dynamics in Molecular Systems (Wiley-VCH, Weinheim, 2011).

[2] J. O. Hirschfelder, C. F. Curtiss, and R. B. Bird, Molecular Theory of Gases and Liquids, second edition (Wiley, New-York, 1964).

[3] I. D. Boyd, and T. E. Schwartzentruber, Nonequilibrium Gas Dynamics and Molecular Simulation (Cambridge University Press, Cambridge, 2017).

[4] J.-M. Hartmann, C. Boulet, and D. Robert, Collisional Effects on Molecular Spectra: Laboratory experiments and models, consequences for applications (Elsevier, Amsterdam, 2008).

[5] N. Owschimikow, F. Königsmann, J. Maurer, P. Giese, A. Ott, B. Schmidt, and N. Schwentner, J. Chem. Phys. 133, 044311 (2010).

[6] J.-M. Hartmann, C. Boulet, H. Zhang, F. Billard, O. Faucher, and B. Lavorel, J. Chem. Phys. 149, 214305 (2018).

[7] Ian F. Tenney, Maxim Artamonov, Tamar Seideman, and Philip H. Bucksbaum, Phys. Rev. A 93, 013421 (2016).

[8] S. Ramakrishna and T. Seideman, Phys. Rev. Lett. 95, 113001 (2005).

[9] J.-M. Hartmann, C. Boulet, T. Vieillard, F. Chaussard, F. Billard, O. Faucher, and B. Lavorel, J. Chem. Phys. 139, 024306 (2013).

[10] T. Vieillard, F. Chaussard, F. Billard, D. Sugny, O. Faucher, S. Ivanov, J.M. Hartmann, C. Boulet, and B. Lavorel, Phys. Rev. A 87, 023409 (2013).

[11] G. Karras, E. Hertz, F. Billard, B. Lavorel, J. M. Hartmann, O. Faucher, E. Gershnabel, Y. Prior, and I. S. Averbukh, Phys. Rev. Lett. 114, 153601 (2015).

[12] G. Karras, E. Hertz, F. Billard, B. Lavorel, G. Siour, J.M. Hartmann, O. Faucher, E. Gershnabel, Y. Prior, and I. S. Averbukh, Phys. Rev. A 94, 033404 (2016).

[13] K. Lin, P. Lu, J. Ma, X. Gong, Q. Song, Q. Ji, W. Zhang, H. Zeng, J. Wu, G. Karras et al., Phys. Rev. X 6, 041056 (2016).

[14] K. Lin, J. Ma, X. Gong, Q. Song, Q. Ji, W. Zhang, H. Li, P. Lu, H. Li, H. Zeng et al., Opt. Express 25, 24917 (2017).

[15] D. Rosenberg, R. Damari, S. Kallush, and S. Fleischer, J. Phys. Chem. Lett. 8, 
$5128(2017)$.

[16] D. Rosenberg, R. Damari, and S. Fleischer, Phys. Rev. Lett. 121, 234101 (2018).

[17] B. Wang, L. He, Y. He, Y. Zhang, R. Shao, P. Lan, P. Lu, Opt. Express 27, 30172 (2019).

[18] H. Zhang, B. Lavorel, F. Billard, J.-M. Hartmann, E. Hertz, O. Faucher, J. Ma, J.

Wu, E. Gershnabel, Y. Prior, and I. S. Averbukh, Phys. Rev. Lett. 122, 193401 (2019).

[19] S. Ramakrishna and T. Seideman, J. Chem. Phys. 124, 034101 (2006).

[20] S. Fleischer, Y. Zhou, R. W. Field, and K. A. Nelson, Phys. Rev. Lett. 107, 163603 (2011).

[21] Matthew J. Murray, Hannah M. Ogden, and Amy S. Mullin, J. Chem. Phys. 148, 084310 (2018).

[22] H. Zhang, F. Billard, X. Yu, O. Faucher, and B. Lavorel, J. Chem. Phys. 148, 124303 (2018).

[23] D. Pentlehner, J. H. Nielsen, A. Slenczka, K. Mølmer, and H. Stapelfeldt, Phys. Rev. Lett. 110, 093002 (2013).

[24] J.-M. Hartmann, C. Boulet, H. Zhang, F. Billard, O. Faucher, B. Lavorel, J. Chem. Phys. 149, 154301 (2018).

[25] J.-M. Hartmann, J. Ma, T. Delahaye, F. Billard, E. Hertz, J. Wu, B. Lavorel, C. Boulet, O. Faucher, arXiv:2002.01212 (2020).

[26] R. Hellmann, J. Chem. Eng. Data 63, 470 (2018)

[27] U. A. Higgoda, R. Hellmann, T. M. Koller, A. P. Froba, Fluid Phase Equilibria 496, 69 (2019)

[28] U. A. Higgoda, R. Hellmann, T. M. Koller, A. P. Froba, Fluid Phase Equilibria 481, 15 (2019)

[29] M. Jeziorska, W. Cencek, K. Patkowski, B. Jeziorski, and K. Szalewicz, J. Chem. Phys. 127, 124303 (2007).

[30] J. Ma, H. Zhang, B. Lavorel, F. Billard, E. Hertz, J. Wu, C. Boulet, J.-M. Hartmann, and O. Faucher, Nat. Commun. 10, 5780 (2019).

[31] K. Lin, I. Tutunnikov, J. Qiang, J. Ma, Q. Song, Q. Ji, W. Zhang, H. Li, F. Sun, X. 
Gong, H. Li, P. Lu, H. Zeng, Y. Prior, I. Averbukh, and J. Wu, Nat. Commun. 9, 5134 (2018).

[32] J. J. Omiste, and L.B. Madsen, Phys. Rev. A, 95023402 (2017).

[33] B. Shepperson, A. A. Søndergaard, L. Christiansen, J. Kaczmarczyk, R. E. Zillich, M. Lemeshko, and H. Stapelfeldt, Phys. Rev. Lett. 118, 203203 (2017).

[34] A. S. Chatterley, C. Schouder, L. Christiansen, B. Shepperson, M. H. Rasmussen, and H. Stapelfeldt, Nat. Commun. 10, 133 (2019). 


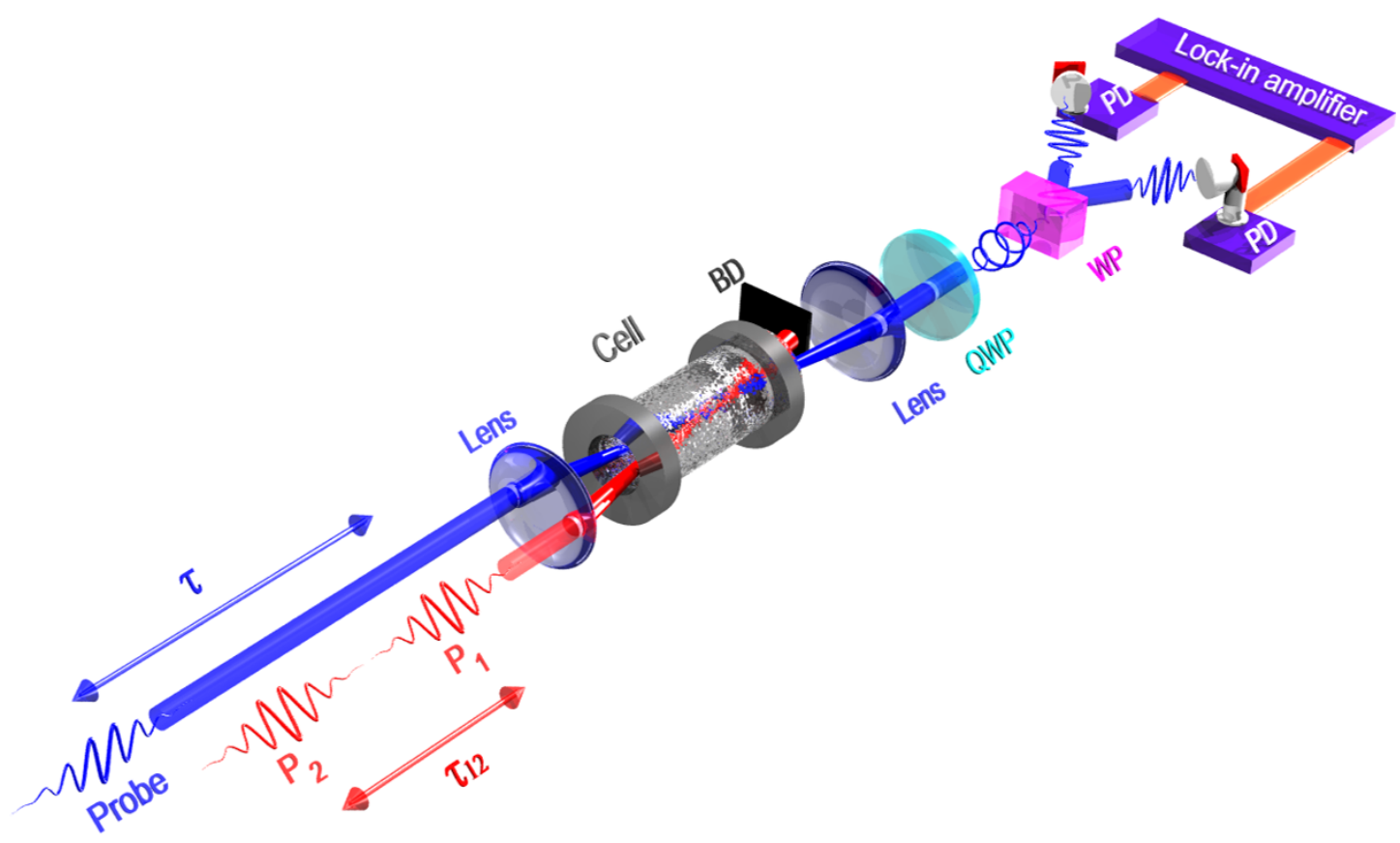

Figure 1 (Color online). Schematic diagram of the rotational alignment echoes created by two time-delayed ultrashort laser pulses $\mathrm{P}_{1}$ and $\mathrm{P}_{2}(800 \mathrm{~nm})$ and probed by a third ultrashort laser pulse $(400 \mathrm{~nm})$. QWP: quarter-wave plate, BD: beam dump, WP: Wollaston prism, PD: photodiode. 

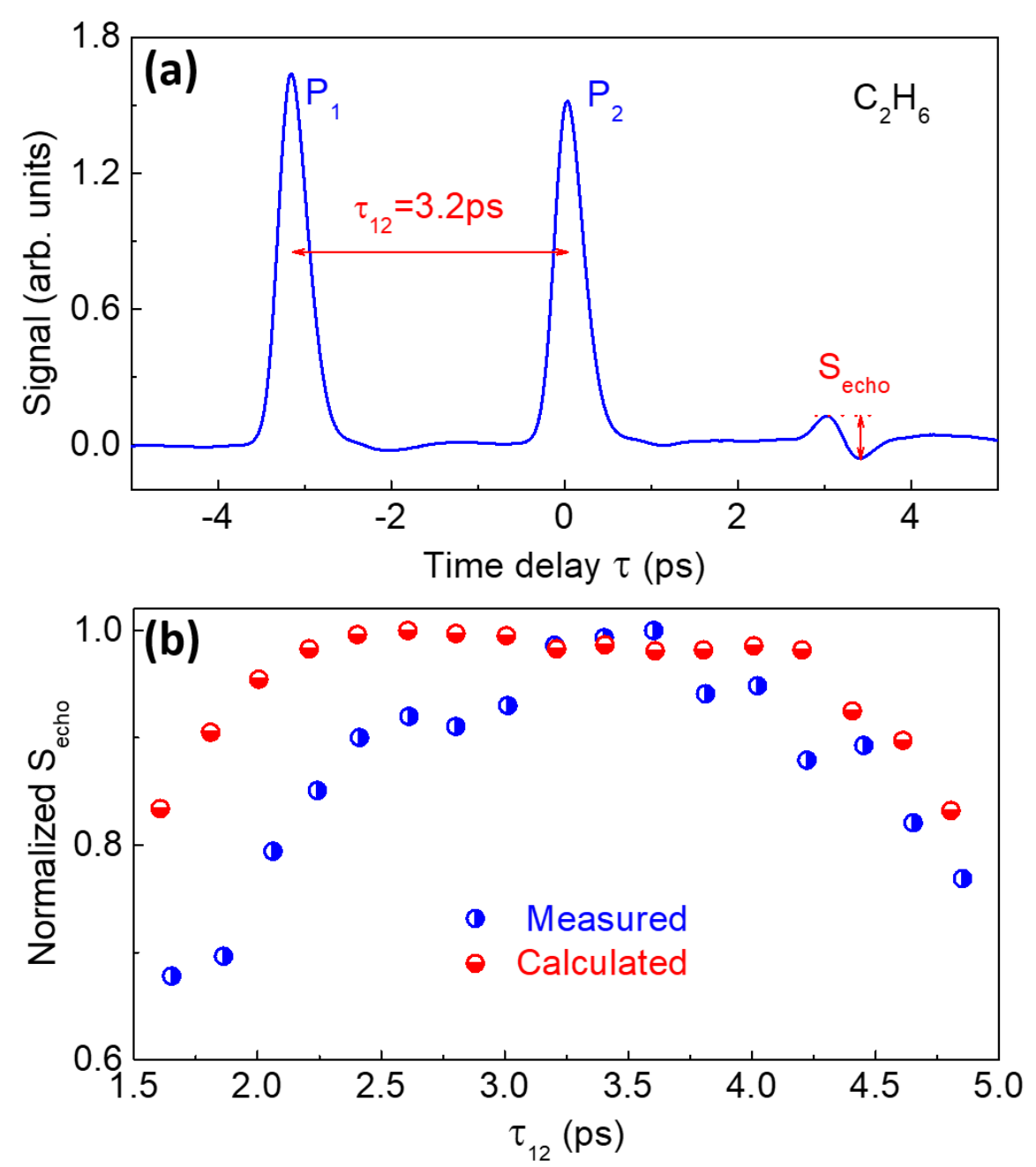

Figure 2 (Color online). (a) Molecular alignment signal measured in $\mathrm{C}_{2} \mathrm{H}_{6}$ gas at low pressure $\left(0.2\right.$ bar). The two laser pulses $P_{1}$ and $P_{2}$ are temporally delayed by $\tau_{12} \sim 3.2$ ps. The echo amplitude $\mathrm{S}_{\text {echo }}$ is measured from peak to dip. (b) Amplitude of the echoes, normalized to its maximum value, measured and calculated in pure $\mathrm{C}_{2} \mathrm{H}_{6}$ for different delays $\tau_{12}$. The intensities of $P_{1}$ and $P_{2}$ in the calculation are set to 40 and 28 $\mathrm{TW} / \mathrm{cm}^{2}$, respectively. 

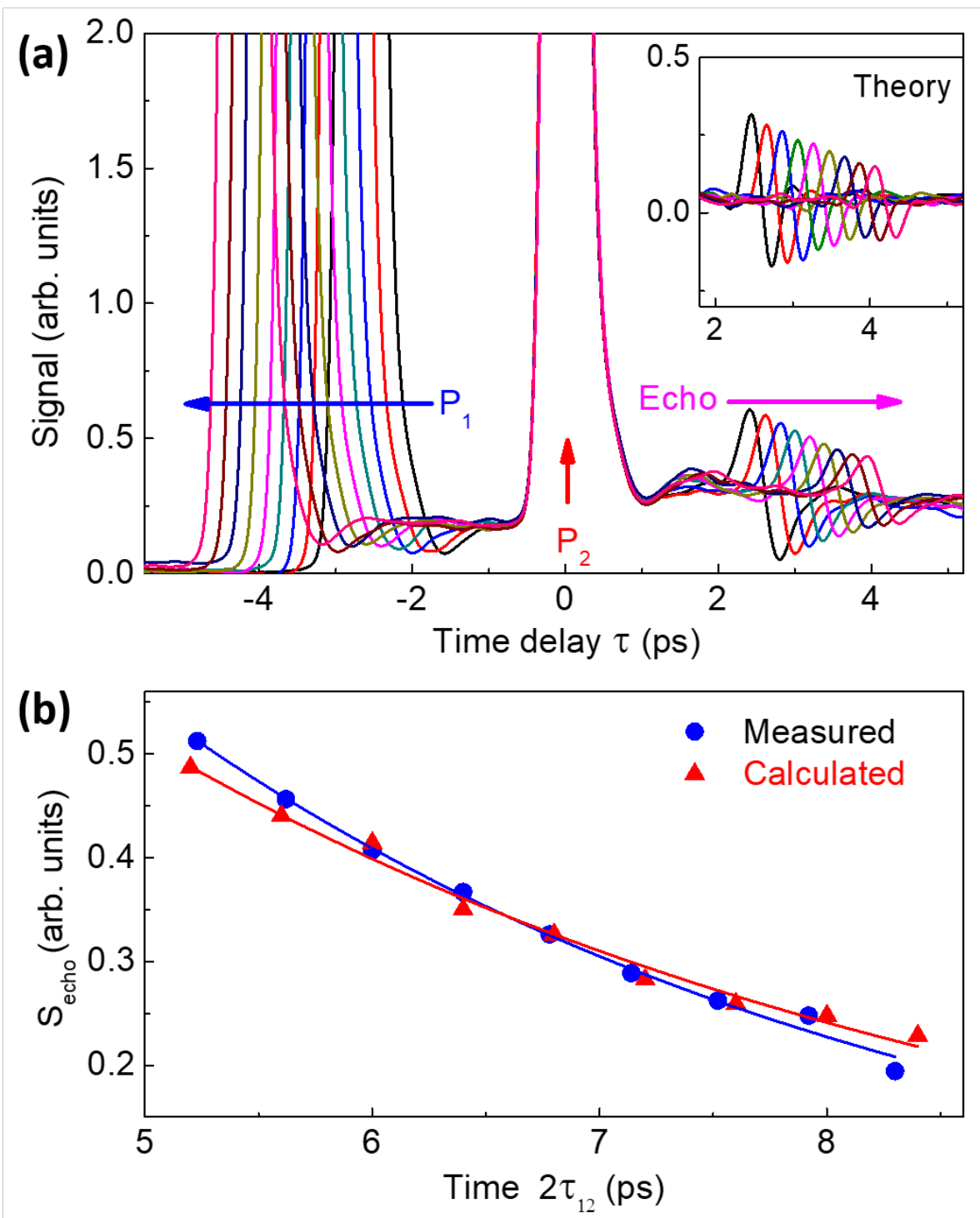

Figure 3 (Color online). (a) High-pressure temporal traces of the echo signal recorded in $\mathrm{C}_{2} \mathrm{H}_{6}$ diluted in $10 \%$ of helium at a pressure of 17 bar. The results of rCMDS calculations performed in the same experimental conditions are displayed in the inset. (b) Measured (blue filled circles) and calculated (red filled triangles) echo amplitude $S_{\text {echo }}$ extracted from (a) as a function of $2 \tau_{12}$ and the exponential fits (solid lines) of the data. 

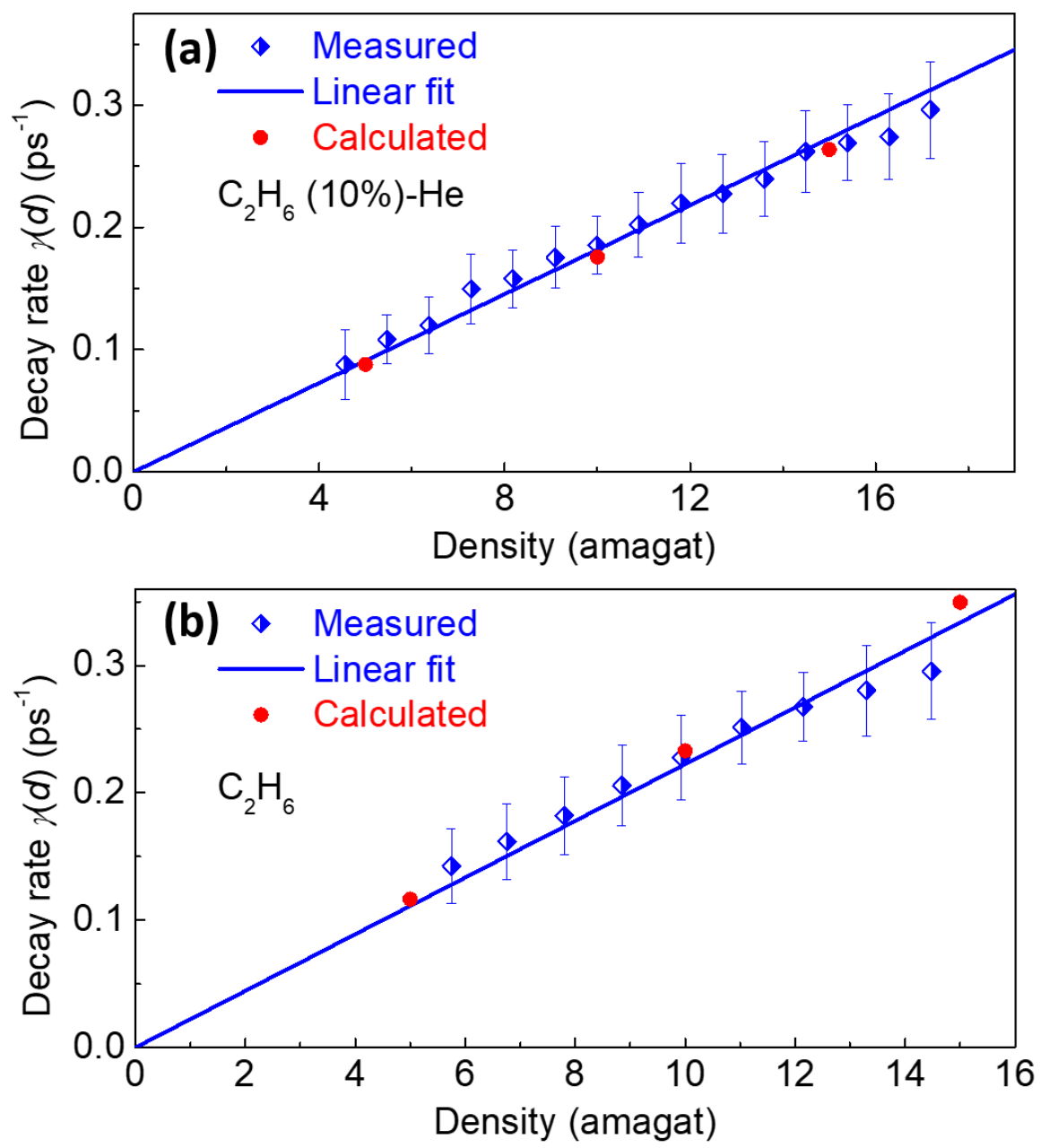

Figure 4 (Color online). (a) Measured (blue diamonds) and calculated (red filled circles) decay rate $\gamma(d)$ of the rotational alignment echo as a function of the density of the $\mathrm{C}_{2} \mathrm{H}_{6}(10 \%)$-He gas mixture and the linear fit (red solid line) applied to the experimental data. (b) Same as (a) but for pure $\mathrm{C}_{2} \mathrm{H}_{6}$ gas. 